\title{
Formation of Gold Nanoprisms on Atomically Flat Mica Surface
}

\author{
Borodinova $\mathrm{TI}^{* 1}$, Styopkin $\mathrm{VI}^{2}$, Kutsenko $\mathrm{VE}^{2}$, Vasko $\mathrm{AA}^{2}$, and Marchenko $\mathrm{OA}^{2}$
}

${ }^{1}$ F.D. Ovcharenko Institute of Biocolloid Chemistry, NAS of Ukraine, 42, Acad. Vernadsky Blvd., 03680 Kyiv, Ukraine ${ }^{2}$ Institute of Physics, NAS of Ukraine, 46 Nauky prosp., 03028, Kyiv, Ukraine

${ }^{*}$ Corresponding author: Borodinova TI, F.D. Ovcharenko Institute of Biocolloid Chemistry, NAS of Ukraine, 42, Acad. Vernadsky Blvd., 03680 Kyiv, Ukraine, E-mail: borodinova@ua.fm

Citation: Borodinova TI, Styopkin VI, Kutsenko VE, Vasko AA, Marchenko OA (2017) Formation of Gold Nanoprisms on Atomically Flat Mica Surface. J Mater Sci Nanotechnol 6(2): 204

Received Date: April 13, 2018 Accepted Date: June 16, 2018 Published Date: June 22, 2018

\begin{abstract}
A numerous methods are being used for synthesis of gold nanoparticles (NPs) in liquid media, in micelles and at different interfaces. The size and geometry of NPs depend on parameters of synthesis. Here we propose the growth of Au NPs on atomically flat mica surfaces. In such conditions the monocrystalline and isotropic NPs are being simultaneously formed on the surface. We have determined the parameters of synthesis at which preferential formation of monocrystalline prism-like nanoparticles (NPRs) is being realized. The percentage of NPRs with triangular and hexagonal habit depends on the surface conditions, in particular, on wettability of the surface. We demonstrate that the size and morphology of grown NPs can be controlled by components of growth medium and parameters of synthesis.
\end{abstract}

Keywords: Nanoparticles; Nanoprisms; Surface wetting angle; Polyvinylpyrrolidone; Ethanol; Ethylene glycol; Glycerol

List of Abbreviations: NPs: Nanoparticles, NPRs: Nanoprisms; SWA: Surface wetting angle; PVP: Polyvinylpyrrolidone; ET: Ethanol; EG: Ethylene glycol; G: Glycerol

\section{Introduction}

Gold nanoparticles (NPs) has received considerable research interest in last decades. Due to unique physical and chemical properties Au NPs are widely used in medicine [1-6], in optoelectronics [7-13], catalysis [14-16], STM and SERS researches [17-20]. Most of studies are focused on the synthesis of gold NPs in aqueous and non-aqueous media, in the micelles and at the phase interfaces. Considerably less attention has received the formation of monocrystalline NPs with regular shape (so-called "nanoprisms", NPRs) on solid surfaces. The authors of reported growth of gold nanowires and nanoplates in aqueous media in presence of cetyltrimethylammonuim bromide on the glass surface coated by indium tin oxide [18]. In [21, 22] gold NPRs have been synthesized on solid substrates ( $\mathrm{Si}$, glass, stainless steel, polyimide, polydimethylsiloxane (PDMS), mica, graphite etc.) using a single step thermolysis of $\left(\mathrm{AuCl}_{4}\right)^{-}{ }^{-}$-tetraoctylammonium bromide complex in air.

To obtain Au NPRs one - and multistage methods of synthesis are used. Nucleus method of NPRs formation was proposed in [23-25] and modified in [26-27]. Therefore, all stages can be summarised as: formation of nuclei $\rightarrow$ changing the conditions of the growth medium $\rightarrow$ adding nuclei to growth medium $\rightarrow$ formation of NPRs. However, result of such synthesis is a mixture of spherical and anisotropic nanostructures that need additional separation.

Among one-stage methods one can distinguish biosynthesis, photoreduction, thermal method, polyol synthesis. Products of the vital activity of microorganisms [28-29], extracts of plants [30], fungi [31] are used as reductions of Au ions and stabilizers of NPR's during biosynthesis. Photoreduction requires presence of photocatalyst, stabilizer and a substance that is an electron donor in a growth medium [32]. Thermal and polyol methods allow to control NPRs in wide range of sizes via (i) changing the ratio of precursor/stabilizer concentration, (ii) the synthesis time and (iii) temperature [33]. All mentioned methods do not require high temperatures except polyol $\left(120-195^{\circ} \mathrm{C}\right)$. In this synthesis EG is used as a dispersion medium and reducer of Au ions, PVP or mixture of PVP+CTAB [34-38] - as stabilizer.

In [38] the flat Au nanocrystals with triangular and hexagonal shape and thickness less than $100 \mathrm{~nm}$ formed in the free volume of the mixture ethanol, ethylene glycol, $\mathrm{HAuCl}_{4}$ (a precursor of gold) and PVP at $80^{\circ} \mathrm{C}$ via polyol method. We propose modified polyol method by adding glycerol. The results of our experiments have demonstrated that immersing of freshly cleaved mica plates into the growth medium drastically changes the character of growth. In such conditions the gold NPRs and NPs are being simultaneously formed on mica surface. Both adding glycerin to the solution and increasing of duration of synthesis lead to 
preferential formation of NPRs. The influence of components in the growth medium on the formation of gold nanostructures on freshly cleaved atomically flat mica substrate was carried out by surface wetting angle (SWA) measurements.

The purpose of this paper is to investigate the growth of Au nanostructures on atomically flat surface of mica immersed in the growth medium of different composition.

\section{Materials and Methods}

To synthesize gold NPRs on the freshly cleaved surface of mica chloroauric acid $\left(\mathrm{HAuCl}_{4} \cdot 3 \mathrm{H}_{2} \mathrm{O}\right.$, "Shanghai Synnad Fine Chemical Co., LTD"), ethyl alcohol, polyvinyl pyrrolidone (29 kDa, "Sigma-Aldrich"), ethylene glycol ("Reahim ", Russia) and glycerin ("Sigma-Aldrich") were used.

The morphology of obtained gold nanostructures and chemical composition were studied by scanning electron microscope (JSM6060), scanning electron microscopy (SEM) (X-ray microanalyzer JXA-733 with energy (EDS) X-ray spectrometer). Estimation of the chemical composition via the EDS was performed with the following parameters and analytical characteristics: accelerating voltage of $20 \mathrm{kV}$; beam current $20 \mathrm{nA}$; spectral resolution $140 \mathrm{eV}$ at line $\mathrm{MnK}$, spatial resolution - 1-5 microns. The reference samples were: $\mathrm{Si}, \mathrm{Al}, \mathrm{Mg}, \mathrm{FeO}\left(\mathrm{SiO}_{2}, \mathrm{Al}_{2} \mathrm{O}_{3}, \mathrm{MgO}, \mathrm{FeO}\right.$, biotite), $\mathrm{Mn}$ (metal Mn), $\mathrm{Ti}$ (ilmenite), $\mathrm{Au}(\mathrm{Au})$; recalculations method $\mathrm{ZAF}$ (atomic number of the $\mathrm{Z}, \mathrm{A}$ absorption, fluorescence $\mathrm{F}$ ), detection limit $-0.01-0.1 \%$.

The influence of components in the growth medium on the formation of gold nanostructures on freshly cleaved atomically flat mica substrate was carried out by surface wetting angle (SWA) measurements. The droplet of each component was deposited on the freshly cleaved bare mica surface and on annealed $\mathrm{Au}(111)$ surface. The volume of deposited droplets was equal to $2 \mu \mathrm{L}$. The value of the contact angle was averaged on the basis of 5 measurements. The error of measured wetting angle was within $\pm 5^{\circ}$.

\section{Results}

\section{Synthesis I}

$15 \mathrm{ml}$ of ethylene glycol (EG) was mixed with $1.6 \mathrm{ml}$ of ethanol (ET). Than $2 \mathrm{ml}$ of PVP C=0,45M (molar concentration per polymer repeating unit) and $1.4 \mathrm{ml}$ of $\mathrm{HAuCl}_{4}$ solution $\left(\mathrm{CAu}=1600 \mathrm{mg} / \mathrm{dm}^{3}\right)$ were added. A freshly cleaved mica plates $(10 \mathrm{~mm} \times 6 \mathrm{~mm})$ were placed vertically in the glass tubes with $5 \mathrm{ml}$ of growth medium. Formation of gold nanostructures was held during 24 hours at $80^{\circ}$ C. The obtained crystals have triangular and hexagonal shape with the lateral dimensions from 30nm up to 20 microns depending on the ratio concentrations of $\mathrm{HAuCl}_{4}, \mathrm{PVP}$ and the time of synthesis. Such conditions contribute to reduction of Au ions via ET, while EG led to reduction at higher temperatures - $120 \div 165^{\circ} \mathrm{C}[39,40]$. The time of synthesis was varied up to 24 hours.

Immersing of mica plate into the growth medium changed the conditions of nucleation. Nucleation occurs in free volume (homogeneous nucleation) as well as on the surface of mica (heterogeneous nucleation). Percentage of NPRs depends on surface conditions, in particular, wettability determined by growth medium. The results of SWA measurements are presented in Table 1.

\begin{tabular}{|c|c|c|c|}
\hline \multirow{2}{*}{\multicolumn{2}{|c|}{ Component }} & \multicolumn{2}{c|}{ Surface wetting angle $\boldsymbol{\theta}^{\circ}$} \\
\cline { 3 - 4 } & $\mathrm{Au}(\mathbf{1 1 1})$ & Mica \\
\hline 1 & EG & 38 & 30 \\
\hline 2 & ET & $<9$ & $<9$ \\
\hline 3 & ET:EG(1:3) & $<9$ & 19 \\
\hline 4 & ET:EG(1:3), PVP & $<9$ & 41 \\
\hline 5 & $\begin{array}{c}\text { ET:EG(1:3), PVP. } \\
\text { HAuCl }\end{array}$ & $<9$ & 17 \\
\hline
\end{tabular}

Table 1: Surface wetting angle according to the composition of the growth medium ET: EG (1:3)

The contact angle of EG with mica surface is $\theta=30^{\circ}$, while ET droplet completely spreads on the surface. Adding PVP up to $0,045 \mathrm{M}$ concentration in the mixture ET+EG changes the contact angle more than 2 times-up to $41^{\circ}$ (against $19^{\circ}$ without PVP, see Table 1). It can be due to adsorption of PVP on mica surface. PVP molecules attach to the substrate via pyrrolidone ( $\gamma$-Lactam) ring of linear polymer units while - $\mathrm{OH}$ groups are located in the opposite direction.

Adding of $\mathrm{HAuCl}_{4}$ in growth medium $(0.5 \mathrm{mM})$ does not change the wetting of $\mathrm{Au}(111)$ surface while the wetting angle for bare mica drops down up to $17^{\circ}$. Thus, the surface of the gold film is completely wetted by growth medium (row 2-5 for Au (111) in table 1), ie., attraction between $\mathrm{Au}$ atoms and components of growth medium is sufficiently strong.

Exposition of mica plate into growth medium (see row 5, Table 1) leads to the formation of gold nanostructures on the surface during 24 hours. As can be seen in SEM images (Figure 1) the surface of a freshly cleaved mica is preferentially covered by aggregates of $\mathrm{Au}$ NPs. The shape of the most nanoparticles is nearly spherical. The particles are covered by thin film of PVP stabilizer (gray «aura» around NPs, Figure 1b-d). The adsorbed film prevents coalescence of NPs. However, the particles are self- 
assembled into separate areas. Aggregate of NPs is depicted in Figure 1d with tilt of substrate to the direction of electron beam at $60^{\circ}$. Flat NPRs with triangular and hexagonal habit rarely appear as well as nanowires (Figure 1c, right conner).

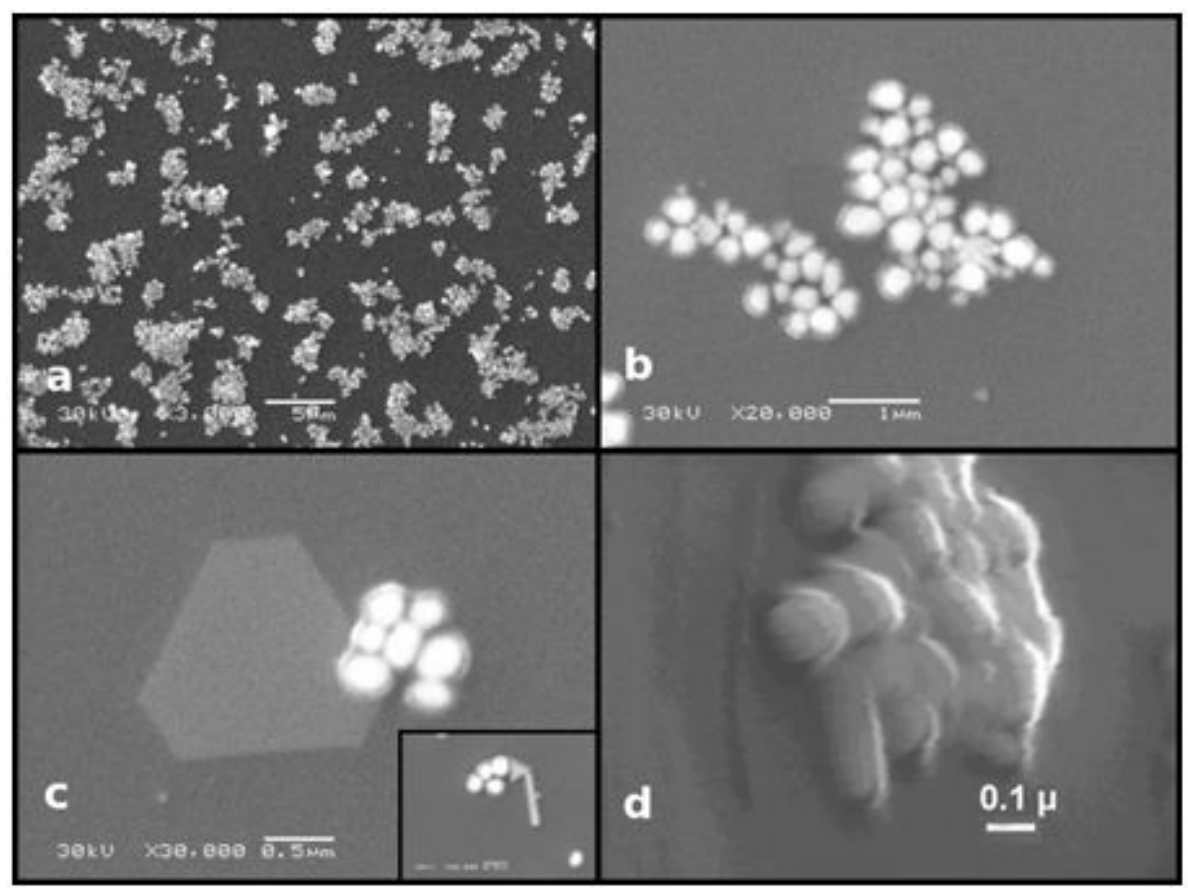

Figure 1: SEM images of gold nanoparticles on mica surface. The growth medium - ET: EG (1:3); $0.5 \mathrm{mM}$ HAuCl4; 0,045M PVP; time of synthesis $\mathrm{t}=24$ hours

\section{Synthesis II}

In order to increase the number of gold NPRs per unit area the conditions of synthesis were changed: (i) in growth media glycerol $\mathrm{G}$ is added, (ii) concentration of gold and synthesis time ( $\mathrm{t}=48 \mathrm{~h})$ is increased. The growth medium contained ET:EG:G (6:7:7), $\mathrm{HAuCl}_{4}(1 \mathrm{mM})$ and PVP $(0,045 \mathrm{M})$. Adding of $\mathrm{G}$ increases viscosity of dispersion medium. Table 2 demonstrates the dynamic viscosity. The value of $\eta_{\mathrm{G}}$ is an order of magnitude greater than $\eta_{\mathrm{EG}}$ and exceeds $\eta_{\mathrm{ET}}$ more than 70 times Table 2 .

\begin{tabular}{|c|c|c|}
\hline Alcohol & Dynamic viscosity $\boldsymbol{\eta}, \mathbf{1 0 - 3 P a} \cdot \mathbf{s}$ & Alcohol \\
\hline ET & $1,2\left(20^{\circ} \mathrm{C}\right)$ & $0,5\left(70^{\circ} \mathrm{C}\right)$ \\
\hline EG & $19,9\left(20^{\circ} \mathrm{C}\right)$ & $3,2\left(80^{\circ} \mathrm{C}\right)$ \\
\hline G & $1480\left(20^{\circ} \mathrm{C}\right)$ & $35\left(80^{\circ} \mathrm{C}\right)$ \\
\hline
\end{tabular}

Table 2: Temperature dependence of surface tension and dynamic coefficient of viscosity of alcohols

However, adding of $\mathrm{G}$ enhances wettability of mica surface. Table 3 shows the results of SWA measurements for mica surfaces and annealed $\mathrm{Au}(111)$.

\begin{tabular}{|c|c|c|c|}
\hline \multicolumn{2}{|c|}{ Component } & \multicolumn{2}{c|}{ Surface wetting angle $\boldsymbol{\theta}{ }^{\circ}$} \\
\cline { 2 - 4 } & Gu(11) & Mica \\
\hline 1 & EG & 37 & 21 \\
\hline 2 & ET:EG:G (6:7:7) & 38 & 30 \\
\hline 3 & $<9$ & $<9$ \\
\hline 4 & ET:EG:G (6:7:7); 0,045M PVP & $<9$ & $<9$ \\
\hline 5 & $\begin{array}{c}\text { ET:EG:G (6:7:7); 0,045M PVP; } \\
\text { 1MM HAuCl }\end{array}$ & $<9$ & $<9$ \\
\hline
\end{tabular}

Table 3: Dependence of wetting angle on composition of the growth medium G:EG:ET (7:7:6)

In contrast to the system of ET:EG(1:3), adding of PVP into a dispersion medium ET:EG:G (6:7:7) increases lyophility of mica surface.

Thus, the redox atoms in growth medium (see Table 3, row 5) are attached much stronger to the mica surface, and the quantity of NPRs is increased. This hypothesis was experimentally confirmed. 
To remove deposited NPs which attached to surface from free volume samples were rinsed in distilled water (Figure 2). Thereafter, we found that NPRs were randomly distributed on the substrate. The most of nanostructures demonstrate regular triangle and hexagonal shape or triangles with truncated vertices.

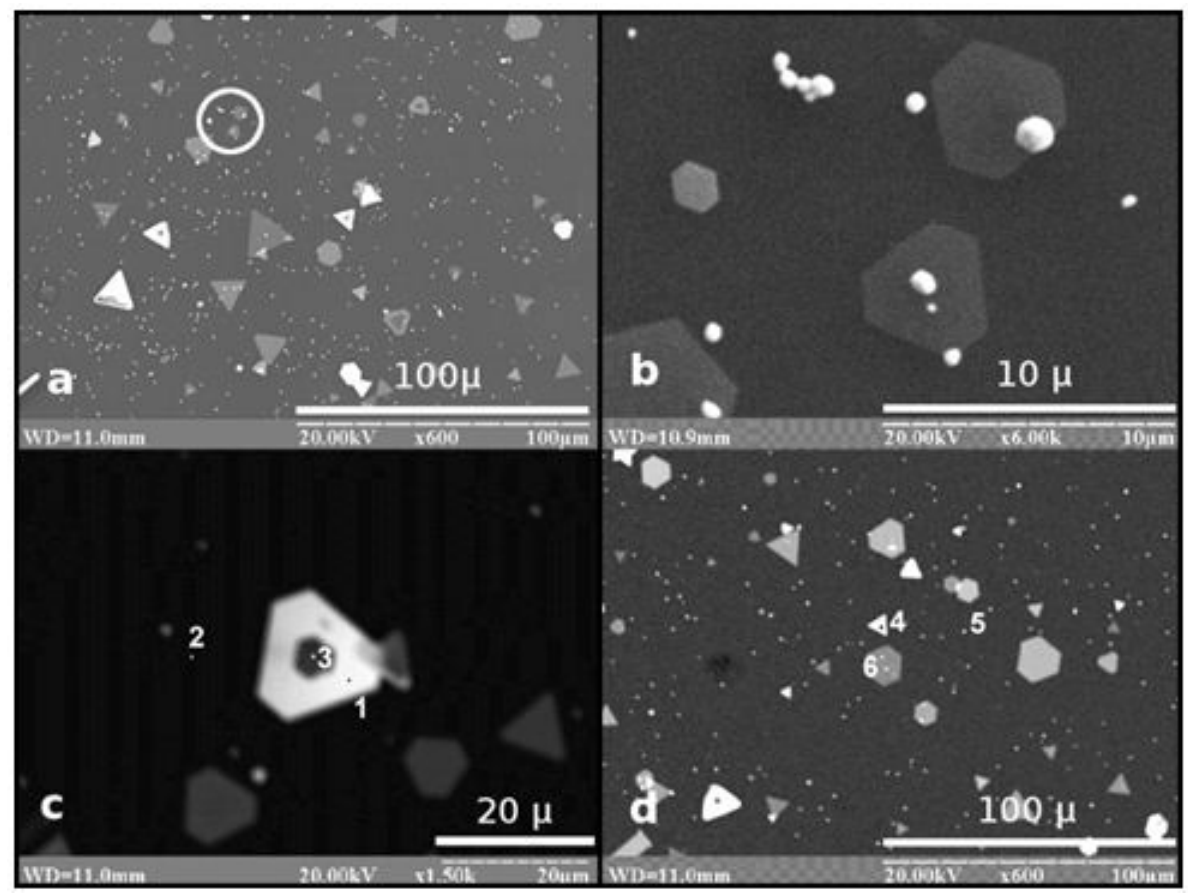

Figure 2: SEM images of gold NPs and NPRs on the surface of mica after rinsing in distilled water. The growth medium: ET: EG: GL (6:7:7); $1 \mathrm{mM} \mathrm{HAuCl}_{4} ; 0,045 \mathrm{M}$ PVP; synthesis time $\mathrm{t}=48$ hours. Numbers denote the points at which the energy dispersive spectra were taken: 1 and 4 - the surface of the thick NPRs, 2 and 5 - mica substrate, 3 - the cavity inside NPRs, 6 - a thin gold NPRs. ${ }^{\star} b$ is an enlarged circular region of image a.

The element composition of the substrate and NPRs was investigated (Table 4). Semi-quantitative microprobe analysis was carried out by EDS. The results in Table 4 are normalized. Analysis shows that the mica substrate is muscovite which includes $\sim 46 \% \mathrm{SiO}_{2}$, $\sim 34 \% \mathrm{Al}_{2} \mathrm{O}_{3}$ and minor impurities of $\mathrm{Na}_{2} \mathrm{O}, \mathrm{MgO}, \mathrm{K}_{2} \mathrm{O}, \mathrm{TiO}_{2}$ and $\mathrm{FeO}$ (Table 4, columns 2 and 5).

\begin{tabular}{|c|c|c|c|c|c|c|}
\hline \multirow{2}{*}{ Elemental composition (\%) } & \multicolumn{7}{|c|}{ Point number } \\
\cline { 2 - 7 } & $\mathbf{1}$ & $\mathbf{2}$ & $\mathbf{3}$ & $\mathbf{4}$ & $\mathbf{5}$ & $\mathbf{6}$ \\
\hline $\mathrm{SiO}_{2}$ & 0,00 & 47,10 & 38,28 & 9,98 & 45,93 & 39,12 \\
\hline $\mathrm{TiO}_{2}$ & 0,00 & 0,30 & 0,46 & 0,05 & 0,40 & 0,43 \\
\hline $\mathrm{Al}_{2} \mathrm{O}_{3}$ & 0,00 & 33,30 & 27,46 & 8,23 & 35,88 & 29,02 \\
\hline $\mathrm{FeO}$ & 0,00 & 1,70 & 2,28 & 2,50 & 1,61 & 2,42 \\
\hline $\mathrm{MnO}$ & 0,00 & 0,00 & 0,03 & 0,30 & 0,00 & 0,17 \\
\hline $\mathrm{MgO}$ & 0,00 & 2,30 & 1,82 & 0,75 & 2,21 & 1,85 \\
\hline $\mathrm{Na}_{2} \mathrm{O}$ & 0,00 & 1,50 & 0,00 & 0,00 & 1,51 & 1,14 \\
\hline $\mathrm{K}_{2} \mathrm{O}$ & 0,00 & 13,80 & 16,75 & 13,22 & 12,56 & 15,93 \\
\hline $\mathrm{Au}$ & 100 & - & 12,92 & 64,97 & - & 9,92 \\
\hline $\mathrm{Sum}$ & 100 & 100 & 100 & 100 & 100 & 100 \\
\hline
\end{tabular}

Table 4: Semi-quantitative microprobe analysis of individual points of the mica surface

Energy dispersive spectrum at point 1 indicates $100 \%$ of gold (Table 4, column 1) i.e. investigated nanoprism is formed by gold. The thickness is large enough to prevent penetration of electron beam. Thus, there are no other lines in the spectrum except of intensive $\mathrm{La}(9,7 \mathrm{keV}), \mathrm{L} \beta 1(11,45 \mathrm{keV})$ and weaker lines of gold $\mathrm{Ma}(2,12 \mathrm{keV})$ and $\mathrm{M} \beta(2.2 \mathrm{keV})$ (see support materials).

\section{Discussion}

The growth mechanism of Au NPs and NPRs under conditions of polyol synthesis can be considered within the LaMayer model [41]. At the first stage $\mathrm{AuCl}_{4}$ - ions reduce to AuO. Thereafter, nuclei are formed in the places of maximum concentration of Au0. Every nucleus can decay if its size is less than critical or continue to grow if its size exceed it. In the last stage augmentation in size of NPs is limited by the growth rate and diffusion of atoms on the surface of NPs. Thus, formation of monocrystals is possible while reduction processes pass gradually. 
Journal of Materials Science \& Nanotechnology

The shape of NPs strongly depends on its internal/external defects and adsorption of PVP. In [42] authors identified a single crystalline, planar-twinned, penta-twinned and complex multiply-twinned structures among the nuclei that obtained via the citrate method. Defects in the form of steps, kinks, and stacking-faults were found on the boundaries of nearby nanotubes. They serve as reactive sites for the growth of nanomaterials. The maximum yield of flat hexagonal Au NPRs with plasmon-driven synthesis was obtained in water-methanol-HAuCl${ }_{4}^{-}$and PVP mixtures, which contained the largest number of planar-twinned seeds. This result confirms the theory of Lofton, Sigmund, who proposed a growth mechanism of large planar prisms. According to [43], the twin (111) planes of the Au and Ag nuclei direct the growth of NPs into prisms or plates with a high aspect ratio. The reason for this is the formation of hollow grooves, which are sites of adatom adherence, and, lowering the nucleation energy, lead to the formation of a new atomic layer in this region, i.e, prism grows laterally.

To revealed possible role of Lofton-Sigmund mechanism in formation of flat NPR we used SEM for thorough study of side faces of NPR in figure 3. Concave shape of these faces should give noticeable contrast in the image of different parts of them. We cannot find any NPR with such a contrast in this experiment. Most of NP give image of side that corresponds to 220 face of gold. The example of such a NPR is given in Figure 3a,b.

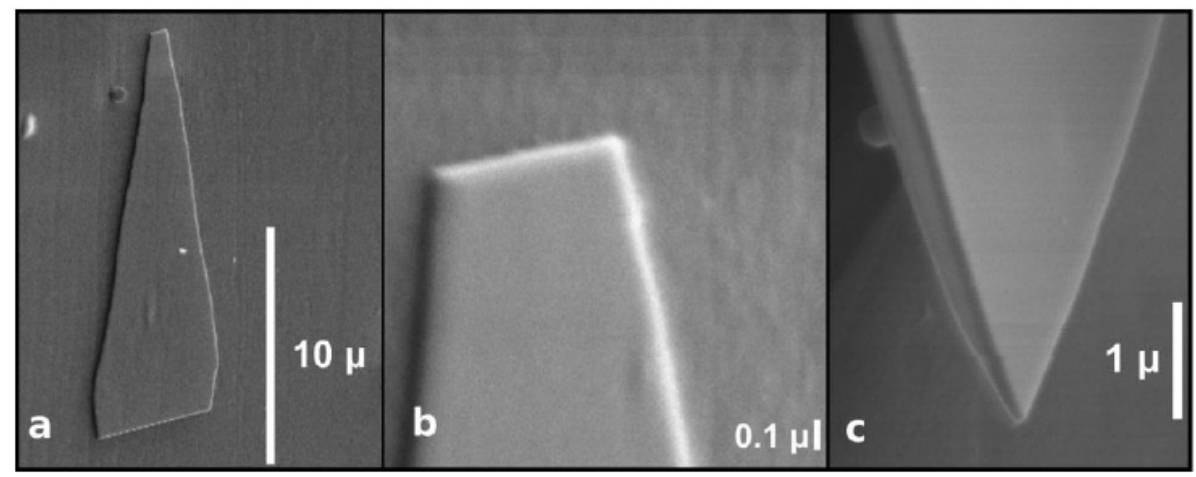

Figure 3: SEM image of hexagonal NPR (a) and part of it (b), (c) concave shape of NPR obtained at tilt of substrate to electron beam of $79^{\circ}$

Only for very rare NPR formed on glass substrate we obtain the image that can serve as an evidence of concave shape of side face. The example of image is shown in figure 3c. One can see a large difference of brightness of upper and low part of side face. This difference may be caused by the different tilt of parts of face to electron beam and corresponds the concave form of the face. Thus on this base we supposed that formation of flat NPR in our experiments is mainly connected with difference of PVP adsorption of gold faces. However, in order to finally establish the mechanism of NPRs formation additional studies are needed.

Equilibrium shape of Au NPs is truncated octahedron [44]. Obtained microprisms with the thickness of tens-hundred nanometers are far from this shape and have a large surface energy. To form such particles with a plate shapes this symmetry has to be broken. Two mechanism of symmetry braking are considered mainly. In [42] and [45] authors supposed presence of twin defects or stacking faults into the nanocrystals. Twin plates composed reentrant grooves that have concave shape thus creating a favorite places for attachment of adatoms.

Another mechanism supposed important role of capping agents (mainly surfactants) in formation of gold nanoplates. What is the role of PVP in formation of Au nanostructures? First, PVP molecules, like other polymers, are adsorbed on the substrate defects, reduce its surface energy, change the surface conditions (see Table 1, line 4). Adsorption of PVP on the substrate determines fixation of AuCl4- ions on it and further formation of nuclei. Molecules of PVP are able to coordinate with $\mathrm{AuCl}_{4}$ - before reduction, forming a complex of PVP / $\mathrm{AuCl}_{4}-[46]$. Therefore, $\mathrm{Au}\left(3^{+}\right)$ions are reduced under more "soft" conditions and form Au clusters of smaller diameter with a narrow size distribution, compared to the system without PVP. An important role in the synthesis of Au NPRs is performed by the pyrrolidone ring [42]. The bond of PVP with Au surface is formed by unshared electron pair of oxygen and nitrogen (pyrrolidone ring) that was confirmed by micro-Raman spectroscopy [38]. Second, a number of researchers indicate [47-49] that PVP molecules are adsorbed on the (111) faces of Au NPRs, blocking their growth in (111) direction. Therefore, growth occurs in lateral direction and thickness of NPRs is in range of tens of nanometers. In [42] Nano SIMS method was used for determining the location of PVP molecules on Au NPRs. It was found that PVP is adsorbed predominantly around the perimeter of NPRs. Even for doubled concentration PVP adsorbed preferentially on the (111) faces. The thickness of NPRs synthesized under such conditions doubled. We work with PVP concentrations in order of magnitude higher than in [42]. Thus, we consider that the adsorption of PVP occurs intensively on both sides of NPR as well as on the flat faces (111).

The adsorbed PVP layer inhibits diffusion of gold atoms to the surface of NPRs, therefore the growth occurs preferentially in the lateral direction. As a result, we observed the flat NPRs $(10 \div 20 \mathrm{~nm}$ thickness) with lateral size up to 10 microns. Finally, PVP molecules adsorbed on the particles serve as protector from their aggregation.

Flat NPRs form via layered and tangential growth mechanism (Kossel-Stranski theory of crystal growth) due to availability of a PVP-stabilizer. As it was shown in [38], uncompleted layers of (111) of NPRs surface form stepped terraces. In Figure 4c and d one can easily see the steps of growth of nanoprisms which correspond to the faces (110) and (100). The gold atoms are being trapped 
by fracture of steps and form uncompleted layer. As the PVP molecules are selectively adsorbed on Au (111), they inhibit the delivery of atoms to the surface and prevent the formation of a new layer of the crystal. Growth of Au NPRs occur mainly in the lateral direction - the crystals become thin and flat (Figure $4 \mathrm{a}$ and b). Thus, NPRs have a maximum surface area (111) and after cleaning from the stabilizer layer can be used as atomically flat substrates for STM [20,50].

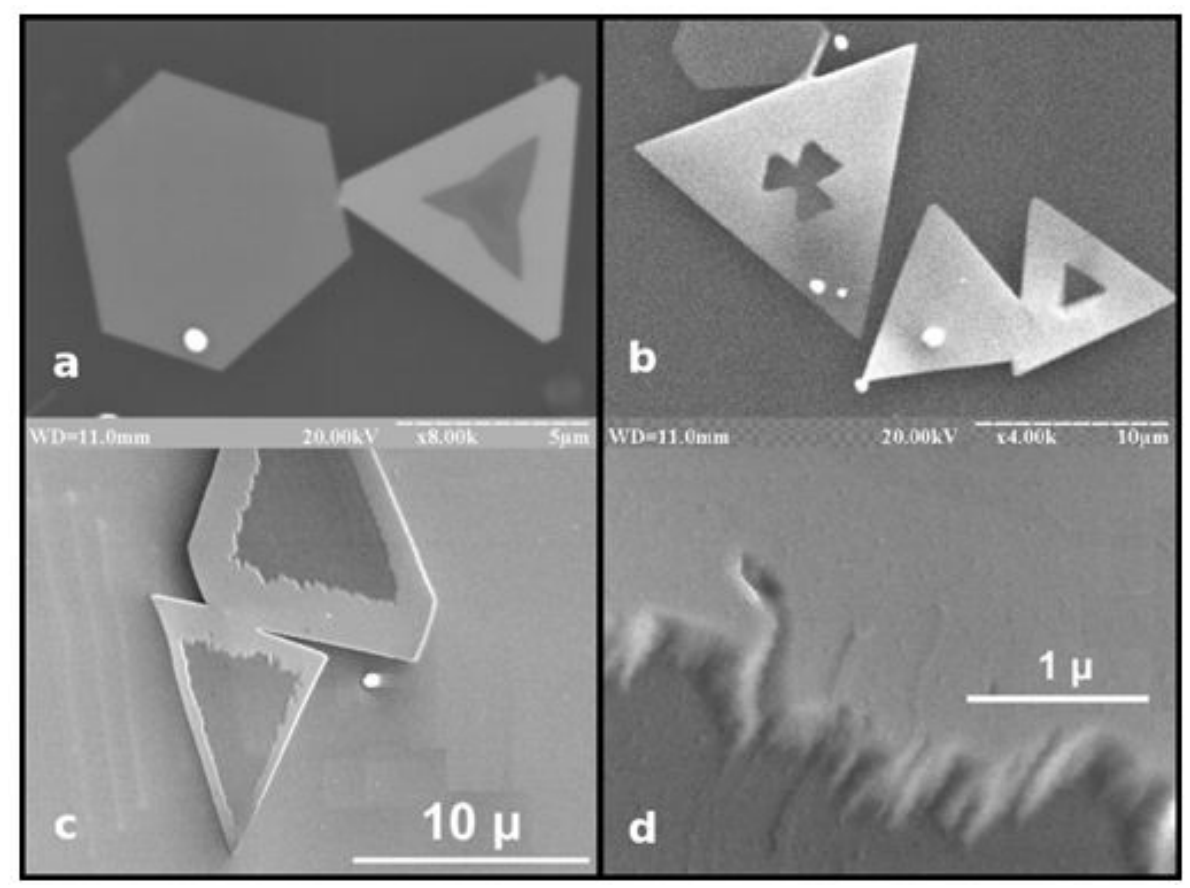

Figure 4: SEM images of the "pits" in nanoprisms

The steps of mica substrate are clearly seen through the two NPRs (Figure 5a). It confirms that the thickness of nanoprisms is in the nanometer range. Extraordinary position of two NPRs is shown in Figure 5b. The lower NPRs has the three petals shape, the upper-a triangle with truncated vertices. It is known that the growth of new atomic layer of the crystal begins at the vertices and edges where the supersaturation of atoms is maximal. Therefore, the formation of dihedral/triangular angle is energetically more favorable. Due to difference in diffusion caused by mutual arrangement of NPRs, the edges of a lower prism growth from vertices to centers. There are also cases when the mutual position of the edges of nanoprisms is parallel (Figure $5 \mathrm{c}$ ). Slit between them is $~$ $300 \mathrm{~nm}$.

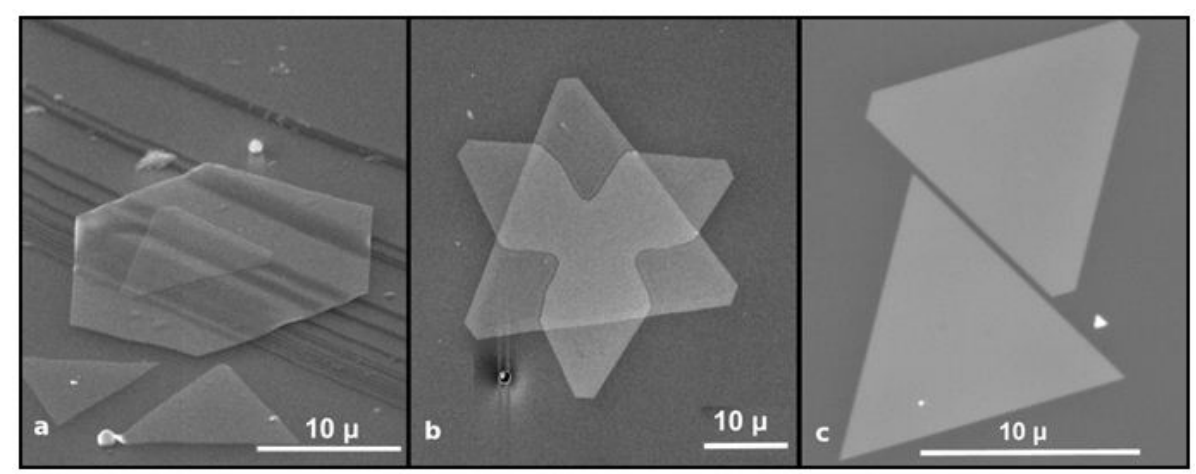

Figure 5: The mutual arrangement of gold nanoprisms on mica surface

\section{Conclusion}

Influence of conditions of synthesis on formation of flat Au nanoprisms on mica surface has been established. It is shown that adding glycerol in the growth medium increases its viscosity. A series of experiments on the surface wetting angle $\theta$ showed that the value $\theta \mathrm{ET}: \mathrm{EG}$ is twice larger than $\theta \mathrm{ET}: \mathrm{EG}: \mathrm{G}$. Thus, a lower value $\theta$ assumes higher wettability. However, adding of PVP into a dispersion medium ET: EG: G (6:7:7) does not make the surface of mica lyophobic in contrast to the system of ET:EG (1:3). Both selective adsorption of PVP molecules on the faces (111) and an increase of viscosity of the growth medium slow the diffusion of gold atoms from the bulk to the surface of the crystal and reduce lateral diffusion along the surface of the growing edge. It causes the formation of flat surfaces of NPRs as well as the presence of holes. Synthesis of Au NPRs on mica substrate is more efficient in the growth medium G:EG:ET (7:7:6) containing 0,045M PVP and $1 \mathrm{mM} \mathrm{HAuCl}_{4}$. At these conditions the number of nanoprisms per unit area of the surface increases. 


\section{Acknowledgment}

The authors acknowledge the resources provided by the F.D. Ovcharenko Institute of Biocolloid Chemistry, NAS of Ukraine, for the realization of this work.

\section{References}

1. Chen PC, Mwakwari SC, Oyelere AK (2008) Gold nanoparticles: From nanomedicine to nanosensing. Nanotechnol Sci Appl 1: 45-66.

2. Jain PK, Huang X, El-Sayed IH, El-Sayed MA (2008) Noble metals on the nanoscale: optical and photothermal properties and some applications in imaging, sensing, biology, and medicine. Acc Chem Res 41: 1578-86.

3. Pekamwar SS, Deshmukh VS, Kalyankar TM (2015) Gold nanoparticles in biomedical applications. Inter Res J Pharm 6: 693-702.

4. Baptista P, Pereira E, Eaton P, Doria G, Miranda A, et al. (2008) Gold nanoparticles for the development of clinical diagnosis methods. Anal Bioanal Chem 391: 943-50.

5. Jianqiang Hu, Zhouping Wang, Jinghong Li (2007) Gold Nanoparticles with Special Shapes: Controlled Synthesis, Surface-enhanced Raman Scattering, and The Application in Biodetection. Sensors (Basel)7: 3299-3311.

6. Huang X, Jain PK, El-Sayed IH, El-Sayed MA (2008) Plasmonic photothermal therapy (PPTT) using gold nanoparticles. Lasers Med Sci 23: 217-28.

7. Luo XL, Xu JJ, Du Y, Chen HY (2004) A glucose biosensor based on chitosan-glucose oxidase-gold nanoparticles biocomposite formed by one-step electrodeposition. Anal Biochem 334: 284-9.

8. Stewart ME, Anderton CR, Thompson LB, Maria J, Gray SK, et.al. (2008) Nanostructured Plasmonic Sensors Chem. Rev. Volume number:108: 494-521.

9. Kern J, Kullock R, Prangsma J, Emmerling M, Kamp M, (2015) et.al. Electrically-driven optical antennas. Nature Photonics $9582-6$.

10. Christian Huck, Andrea Toma, Frank Neubrech, Manohar Chirumamilla, Jochen Vogt, et.al. (2015) Gold Nanoantennas on a Pedestal for Plasmonic Enhancement in the Infrared ACS Photon 2: 497-505.

11. Huang JS, Callegari V, Geisler P, Brüning C, Kern J, et.al. (2010) Atomically flat single-crystalline gold nanostructures for plasmonic nanocircuitry. Nat Commun 1:350-7.

12. Zhengchun Liu, Fengliang Xue, Yi Su, Yuri M Lvov, Kody Varahramyan (2006) Memory Effect of a Polymer Thin-Film Transistor With Self-Assembled Gold Nanoparticles in the Gate Dielectric. IEEE Transac on Nanotechnol 5: 379-84.

13. Raju Kumar Gupta, Gao Ying, Srinivasan MP , Pooi See Lee (2012) Covalent Assembly of Gold Nanoparticles: An Application toward Transistor Memory. J Phys Chem B 116: 9784-90.

14. Xu W, Kong JS, Chen P (2009) Probing the catalytic activity and heterogeneity of Au-nanoparticles at the single-molecule level. Phys Chem Chem Phys 11: 2767-78.

15. Haruta M (2004) Gold as a Novel Catalyst in the 21st Century: Preparation, Working Mechanism and applications. Gold Bulletin 37: 27-36.

16. Masatake H, Masakazu D (2001) Advances in the catalysis of Au nanoparticles. Appl Cataly A General 222: 427-37.

17. Fana M, Andrade GF, Brolo AG (2011) A review on the fabrication of substrates for surface enhanced Raman spectroscopy and their applications in analytical chemistry. Anal Chim Acta 693: 7-25.

18. Borodinova TI, Kravets VG, Romanyuk VR (2012) Gold Nanocrystals as a Substrate for Micro Raman Spectroscopy J Nano- Electron Phys 4: 1-8.

19. Shibu ES, Kimura K, Pradeep T (2009) Gold Nanoparticle Superlattices: Novel Surface Enhanced Raman Scattering Active Substrates. Chem Mater 21:3773-81 20. Dahanayaka DH, Wang JX, Hossain S, Bumm LA (2006) Optically Transparent Au\{111\} Substrates: Flat Gold Nanoparticle Platforms for High-Resolution Scanning Tunneling Microscopy. J Am Chem Soc 128: 6052-3.

21. Sajanlal PR, Pradeep T (2008) Growth of anisotropic gold nanostructures on conducting glass surfaces J Chem Sci 120: 79-85.

22. Radha B, Arif M, Datta R, Kundu T K, Kulkarni GU (2010) Movable Au Microplates as Fluorescence Enhancing Substrates for Live Cells. Nano Res 3: 738-47. 23. Sau TK, Murphy CJ (2004) Room Temperature, High-Yield Synthesis of Multiple Shapes of Gold Nanoparticles in Aqueous Solution. J Am Chem Soc 126: $8648-9$.

24. Millstone JE, Park S, Shuford KL, Qin L, Schatz GC, et.al. (2005) Observation of a Quadrupole Plasmon Mode for a Colloidal Solution of Gold Nanoprisms. J Am Chem Soc 127: 5312-3.

25. Millstone JE, Métraux GS, Mirkin CA (2006) Controlling the edge length of gold nanoprisms via a seed-mediated approach. Adv Func Mater 16: 1209-14.

26. Lee D, Hong S, Park S (2011) Controlled Assembly of Gold Nanoprism and Hexagonal Nanoplate Films for Surface Enhanced Raman Scattering. Bull Korean Chem Soc 32: 3575-80.

27. Scarabelli L, Coronado-Puchau M, Giner-Casares JJ, Langer J, Liz-Marzán LM (2014) Monodisperse Gold Nanotriangles: size control, large-scale self-assembly, and Performance in Surface-Enhanced Raman Scattering. ACS Nano 8: 5833-42.

28. Estrela-Llopis VR, Borodinova TI, Yurkova IN (2010) Extracellular Biomineralization and Synthesis of Gold and Platinum Nano- and Microcrystals in Polysaccharide Aqueous Solutions. In: "Nano-Science: Colloidal and Interfacial Aspects". Ed. V.M.Starov, CPC Press Taylot\&Francis Group, LLC, London, New York 307-368.

29. Ogi T, Saitoh N, Nomura T, Konishi Y (2010) Room-temperature synthesis of gold nanoparticles and nanoplates using Shewanella algae cell extract. J Nanopart Res 12: 2531-39.

30. Shankar SS, Rai A, Ankamwar B, Singh A, Ahmad A, et.al. (2004) Biological synthesis of triangular gold nanoprisms. Natur Mater 3: 482-8).

31. Verma VC, Singh SK, Solanki R, Prakash S (2011) Biofabrication of Anisotropic Gold Nanotriangles Using Extract of Endophytic Aspergillus clavatus as a Dual Functional Reductant and Stabilizer. Nanoscale Res Lett 6:16.

32. Miranda A, Malheiro E, Skiba E, Quaresma P, Carvalho P.A, et.al. (2010) One-pot synthesis of triangular gold nanoplates allowing broad and fine tuning of edge length. Nanoscale 2: 2209-16).

33. Chu H-C, Kuo C-H, Huang MH (2006) Thermal Aqueous Solution Approach for the Synthesis of Triangular and Hexagonal Gold Nanoplates with Three Different Size Ranges. Inorg Chem 45: 808-813.

34. Kim F, Connor S, Song H, Kuykendall T, Yang PD (2004) Platonic gold nanocrystals. Angew Chem Int Edit Engl 43: 3673-7. 
35. Li BC, Cai W, Cao B, Sun F, Li Y, et.al. (2006) Mass Synthesis of Large, Single-Crystal Au Nanosheets Based on a Polyol Process. Adv Funct Mater 16: 83-90. 36. Wang C, Kan C, Zhu J, Zeng X, Wang X, et.al. (2010) Synthesis of High-Yield Gold Nanoplates: Fast Growth Assistant with Binary Surfactants. J Nanomater 2010: 9 .

37. Liuab H, Yang Q (2011) A two-step temperature-raising process to gold nanoplates with optical and surface enhanced Raman spectrum properties. CrystEngComm 13: 2281-8.

38. Borodinova TI, Sapsay VI, Romanyuk VR (2015) Gold Nanocrystals Growth in the Mixture of Primary Alcohols. J Nano- Electron Phys 7: 01032-1-10.

39. Wang C, Kan C, Zhu J, Zeng X, Wang X, et.al. (2010) Synthesis of High-Yield Gold Nanoplates: Fast Growth Assistant with Binary Surfactants. J Nanomater 2010: 9 .

40. Tsuji M, Miyamae N, Hashimoto M, Nishio M, Hikino S, et.al. (2007) Shape and size controlled synthesis of gold nanocrystals using oxidative etching by AuCl4and Cl- anions in microwave-polyol process. Colloids and Surfaces A: Physicochem Engin Aspe 302: 587-8.

41. LaMer VK, Dinegar RH (1950) Theory, Production and Mechanism of Formation of Monodispersed Hydrosols. J Am Chem Soc 72: 4847-54.

42. Zhai Y, DuChene JS, Wang Y-Ch, Qiu J, Johnston-Peck AC, et.al. (2016) Polyvinylpyrrolidone-induced anisotropic growth of gold nanoprisms in plasmondriven synthesis. Nature Mater 15:889-5.

43. Lofton C, Sigmund W (2005) Mechanisms Controlling Crystal Habits of Gold and Silver Colloids. Adv Funct Mater 15: 1197-208.

44. Barnard AS, Lin XM, Curtiss LA (2005) Equilibrium Morphology of Face-Centered Cubic Gold Nanoparticles $>3$ nm and the Shape Changes Induced by Temperature. J Phys Chem B 109: 24465-72.

45. Prof. Younan Xia, Dr. Yujie Xiong, Dr. Byungkwon L, and Dr. Sara ES (2009) Shape-Controlled Synthesis of Metal Nanocrystals: Simple Chemistry Meets Complex Physics? Angew Chem Int Ed Engl 48: 60-103.

46. Yonezawa T, Toshima N (1995) Mechanistic Consideration of Formation of Polymer-protected Nanoscopic Bimetallic Clusters. J Chem Soc Faraday Trans 91: 4111-9.

47. Tsuji M, Hashimoto M, Nishizawa Y, Kubokawa M, Tsuji T (2004) Microwave-assisted Synthesis of Metallic Nanostructures in Solutions. Chem Eur J 11: 440-52. 48. Li C, Cai W, Li Y, Hu J, Liu P (2006) Ultrasonically Induced Au Nanoprisms and Their Size Manipulation Based on Aging. J Phys Chem B 110: 1546-52. 49. Millstone JE, Hurst SJ, Metraux GS, Cutler JI, Mirkin CA (2009) Colloidal Gold and Silver Triangular Nanoprisms. Small 5: 646-64.

50. Dahanayaka DH, Bumm LA (2010) Surface preparation of supported flat gold nanoparticles for use as Au (111) single crystal substrates AVS 57 th International Symposium \& Exhibition-Nanometer-scale Science and Technology.

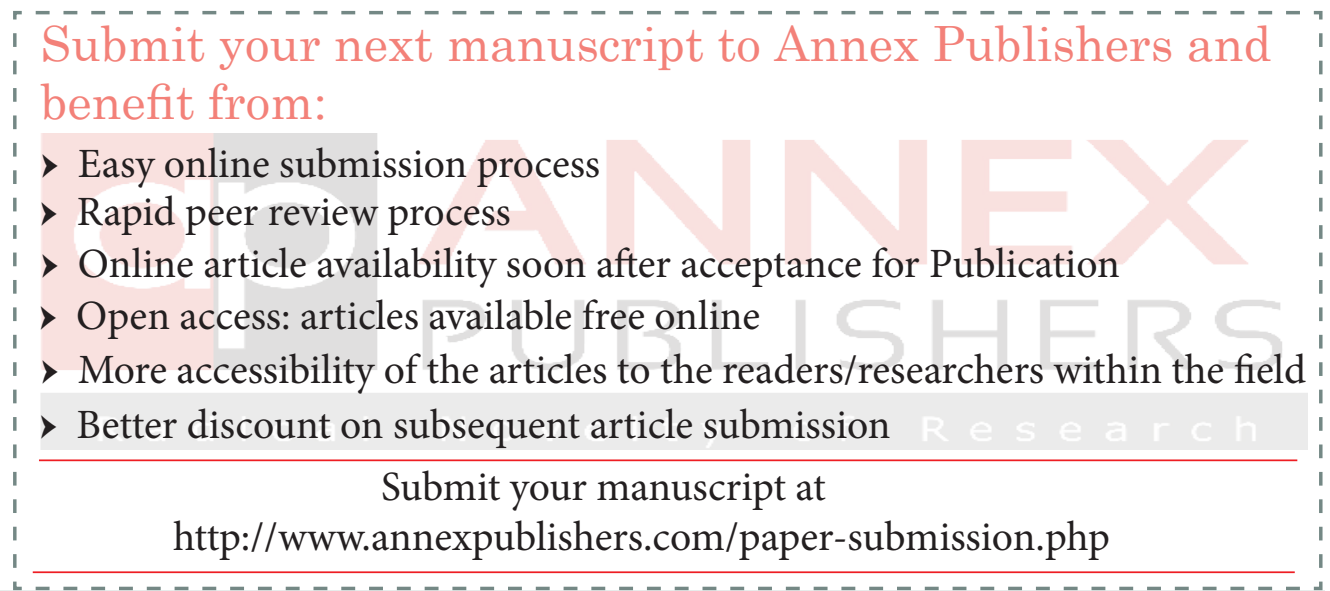

